Sexualität darf kein Tabu sein

\section{Auch geistig Behinderte sehnen sich nach Liebe und Intimität}

\begin{abstract}
Die Themen Liebe und Sexualität werden beim Umgang mit Menschen mit schweren körperlichen und/oder geistigen Behinderungen oft ausgeklammert. Wir haben bei Dr. Peter Martin, Chefarzt der Séguin-Klinik für Menschen mit schwerer geistiger Behinderung am Epilepsiezentrum Kork, nachgefragt, wie man diesen Patienten einen offenen Umgang mit Sexualität ermöglichen kann.
\end{abstract}

MMW: Welche Bedeutung hat Liebe und Sexualität für Menschen mit schweren geistigen Behinderungen?

Martin: Grundsätzlich hat Liebe, Sexualität und Nähe bei Menschen mit Behinderungen den gleichen hohen Stellenwert wie bei Menschen ohne Behinderung. Möglicherweise ist der Stellenwert bei ihnen sogar noch höher einzuschätzen.

MMW: Wo sehen Sie Einschränkungen für körperlich und geistige Behinderte? Martin: Wegen der Grunderkrankung und der Mehrfachbehinderung können natürlich zahlreiche körperliche Einschränkungen bestehen. Dazu können z.B. eine gestörte Pubertätsentwicklung, Fehlbildungen der Geschlechtsorgane oder motorische Störungen wie Zerebralparesen mit Spastik von Armen und Beinen gehören. Häufig besteht auch eine Divergenz zwischen körperlicher und psychischer Reife, die die Sexualität beeinträchtigen kann. Hinzu kommt, dass viele Menschen mit geistigen Behinderungen sich schwer damit tun, soziale Zeichen zu verstehen. Und vielen wurde nie beigebracht, welche Formen der Nähe und Sexualität nur in die Privatsphäre gehören. Dies kann zu Konflikten führen.

Die größten Einschränkungen sind aber meist durch das soziale Umfeld vorgegeben. So leben behinderte Menschen in Wohnheimen auch heute noch oft in 2- und 3-Bett-Zimmern, in denen Betreuer ohne anzuklopfen ein- und ausgehen. Ein privater Rückzugsraum fehlt oft völlig.

MMW: Wie ist die rechtliche Lage - auch in Hinblick auf die Kontrazeption?
Martin: Rechtlich gesehen - und selbstverständlich auch nach meiner vollen Überzeugung - haben auch Menschen mit Behinderungen ein Recht auf Privatsphäre, und der Wunsch nach Sexualität und Partnerschaft muss akzeptiert werden. Auch bei der Frage nach Verhütungsmaßnahmen müssen der Wille und die Entscheidung des Patienten berücksichtigt werden. Ist der Patient nicht in der Lage, die Zusammenhänge zwischen Sexualität, Schwangerschaft und Kontrazeption zu begreifen, muss der gesetzliche Betreuer in seinem Sinne entscheiden. Die Sterilisation von Menschen mit geistiger Behinderung ist nur in Ausnahmen erlaubt. Bei mangelnder Einsichtsfähigkeit reicht nicht die Entscheidung des gesetzlichen Betreuers, sondern es muss ein Sterilisationsbetreuer hinzugezogen werden, und das Vormundschaftsgericht muss zustimmen. Möglich ist die Sterilisa-

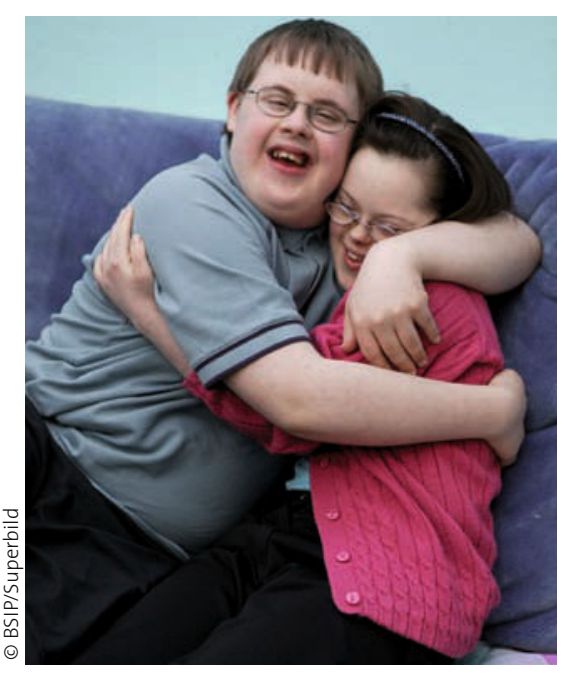

Nähe und Zärtlichkeit sind auch für sie eine Quelle des Glücks.

\section{NACHGEFRAGT}

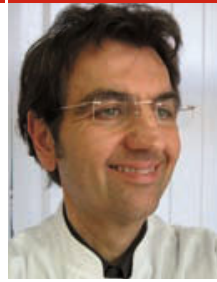

bei Dr. med. P. Martin, Kork

„Oft fehlt ein privater Rückzugsraum, in dem

Sexualität ungestört ausgelebt werden kann." tion nur dann, wenn andernfalls Gefahr für Gesundheit und Leben besteht.

Ein Kinderwunsch geistig behinderter Menschen ist bei uns selbst beifesten Partnerschaften bisher kaum gesellschaftlich akzeptiert. Nur wenige Projekte, z.B. der Lebenshilfe, bieten geistig behinderten Menschen die Möglichkeit, mit ihren Kindern zusammen zu leben.

MMW: Besteht aus Ihrer Sicht auch ein Missbrauchsrisiko?

Martin: Dieses Gebiet ist wenig beforscht - man geht aber davon aus, dass das Missbrauchsrisiko bei behinderten Menschen mindestens doppelt so hoch ist wie bei Nicht-Behinderten. Die Täter sind meist unter Verwandten oder Betreuern zu suchen. Meist handelt es sich um einen wiederholten Missbrauch über Jahre. Bei Schwerstbehinderten kann durch die Notwendigkeit zur Intimpflege die Grenze zur sexuellen Manipulation oft nicht so klar gezogen werden. Um Missverständnissen vorzubeugen, sollte dieses Thema auch mit den Eltern offen diskutiert werden.

MMW: Wie kann man Menschen mit Behinderungen einen möglichst freien Umgang mit Sexualität ermöglichen? Martin: Noch vor Einsetzen der Pubertät sollte auch geistig behinderten Kindern nach Möglichkeit vermittelt werden, welche Geschlechtsunterschiede bestehen und was in der Pubertät auf sie zukommt. Ganz wichtig ist zu vermitteln, was in den Privatbereich gehört und was in der Öffentlichkeit besprochen werden kann. Und die Kinder sollten auch frühzeitig lernen, „nein“ zu sagen, wenn ihnen z.B. die intime Körperpflege zu weit geht.

- Interview: Maria Weiß 